\title{
Calidad, satisfacción y demografía residencial. Una revisión conceptual de enfoques y tensiones de las teorías*
}

\begin{tabular}{|c|c|}
\hline \multicolumn{2}{|c|}{ Fecha de recepción: 15 de mayo de 2013 Fecha de aceptación: 24 de noviembre de 2013 Disponible en línea: 30 de julio de 2014} \\
\hline \multicolumn{2}{|c|}{ Diva Marcela García García } \\
\hline \multicolumn{2}{|c|}{ Magíster en Urbanismo y magíster en Estudios Territoriales y de la Población con mención en Demografía } \\
\hline Profesora Pontificia Universidad Javeriana & marcela.garcia033@gmail.com \\
\hline
\end{tabular}

Resumen El texto explora las posibilidades conceptuales y metodológicas que abre el estudio de la demografía residencial y enfatiza en el estudio de las percepciones de calidad y satisfacción residencial a partir de características particulares de los hogares que las habitan. Desarrolla tres tensiones conceptuales que enriquecen el debate sobre la construcción de entornos residenciales óptimos: población-vivienda, objetivo-subjetivo y vivienda-residencia.

Palabras clave

Demografía; percepción; población; vivienda

\footnotetext{
Artículo de investigación científica y tecnológica. Parte de la memoria de investigación "Calidad de la vivienda y satisfacción residencial en España 2004-2007", desarrollada en la Universitat Autónoma de Barcelona, Departamento de Geografía, Centre d'estudis demografics, en junio de 2011, para optar al título de magíster en Estudios Territoriales y de la población con mención en Demografía.
}

Cómo citar este artículo: García-García, D. M. (2014). Calidad, satisfacción y demografía residencial. Una revisión conceptual a de los enfoques y tensiones de las teorías. Cuadernos de Vivienda y Urbanismo, 7(14), pp. 260-275. http://dx.doi.org/10.11144/Javeriana.CVU7-14.csdr 


\section{Quality, Satisfaction and Residential Demography. A Conceptual Revision to the Approaches and Theories Tensions}

Abstract This text explores the conceptual and methodological possibilities opened by housing demography, emphasizing the study of perceptions of quality and residential satisfaction from characteristics of households. It develops three conceptual tensions that enrich the debate on optimal residential environments: population- housing, objective-subjective, and housing-residence.

Keywords Population; housing; demography; perception

\section{Qualidade, satisfação e demografia} residencial. uma revisão conceptual aos enfoques e tensões das teorias

Resumo Este texto explora as variantes conceptuais e metodológicas que se enquadram no estudo da demografia residencial, com um particular enfoque no estudo das percepçóes da qualidade e da satisfaçáo das pessoas com as suas residências, a partir de determinadas características particulares dos lares onde habitam. Desenvolve, por isso, três tensóes conceptuais que enriquecem o debate sobre a construção de áreas residenciais ótimas: população-vivenda, objetivo-subjetivo e vivenda-residência.

Palavras chave Demografia; percepção; população; vivenda 


\section{Introducción}

El presente trabajo tiene por objeto presentar algunas perspectivas conceptuales que se abren a partir de la aplicación de preceptos y herramientas demográficas a problemáticas del campo de los estudios residenciales. Se pretende situar los estudios sobre calidad y percepción de la vivienda en el ámbito de las disciplinas que estudian las dinámicas residenciales de la población, como un ejemplo de elementos de análisis de máximo interés para la construcción de entornos residenciales óptimos. Este planteamiento tiene como finalidad hacer un llamado de atención en el contexto colombiano sobre la necesidad de no posponerlo importante por lo urgente que, en este caso, se refiere a que aún en un contexto de déficit cuantitativo es fundamental hilvanar apuestas conceptuales que apunten a la satisfacción residencial. Esto solo puede lograrse por la exploración de recursos y planteamientos de las diversas disciplinas que abordan el hecho residencial.

Para ello, se desarrollan cuatro apartados que van de lo general a lo particular. En el primero se aborda el campo de los estudios de vivienda y se identifica su relación con diversas disciplinas, lo cual ha permitido estudiar la vivienda desde una óptica integral correspondiente con su papel dentro del sistema social.

Luego, se hace un acercamiento a la demografía residencial y se reconocen sus principales intereses y postulados. Ese es el punto de partida para explorar los avances en los estudios que se ocupan de la calidad y la satisfacción residencial. Al final se presenta un apartado a manera de síntesis que presenta las tensiones conceptuales que se pretende poner en consideración para la reflexión del contexto residencial colombiano.

\section{El campo de los estudios sociales de la vivienda}

Las definiciones del concepto de vivienda son diversas y en su mayoría corresponden a ópticas de análisis diferentes, con problemáticas de estudio particulares que dan lugar a enfoques disciplinares distintos.

Algunas la describen como un bien necesario, una mercancía, una forma de patrimonio e inversión económica y un lugar para vivir en sociedad. Esta última se toma como punto de partida del presente trabajo, al considerar que la "inserción de la vivienda en la sociedad" es generada, según Cortés (1995), mediante dos vías que confirman su importancia: la primera, relacionada con las prácticas familiares que suceden en su interior; por medio de ellas, las personas aprenden y ensayan los roles familiares básicos, así como los valores y las costumbres dominantes en su entorno cultural. Es allí donde se establecen los límites de la vida pública y la vida privada, diferenciación que forma parte fundamental de la estructura de la vida en sociedad y de las características culturales propias de la misma, ya que incluso "cada civilización inscribe en la vivienda las normas esenciales de su espacio" (Pezeu-Massabau, 1998, citado en Cortés, 1995, p. 41).La segunda vía por la que se identifica el rol de la vivienda en la sociedad tiene en cuenta la escala espacial en la que se inserta y que a la vez 
conforma, si se define como el "tipo de espacio particular y específico que se encuentra insertado en un contexto más general formado por el vecindario, el pueblo o ciudad, un país y una civilización" (Cortés, 1995, p. 38). Lo anterior cobra mayor importancia si se parte del supuesto de que el espacio no es neutral, homogéneo ni unitario, sino que es fruto de las contradicciones y los conflictos de la misma estructura social; por ello, la vivienda misma es un objeto específico de conflicto social, pues está asociada con el lugar que se asigna a cada familia en la sociedad y las potencialidad y restricciones que vienen ligadas a dichas localizaciones.

Por todo lo anterior, a pesar de la diversidad, todas las posturas teóricas actuales confluyen en el reconocimiento de la vivienda como una variable clave en la organización de la sociedad moderna, ya que aborda una dimensión intermedia entre el individuo y la sociedad.

No obstante, los estudios de vivienda no siempre han reconocido el alcance de la misma como hecho social, porque han concentrado sus esfuerzos investigativos en casos particulares, sin integrar dichos estudios en un marco más general que aborde su integración en la estructura social. Tras reconocer este panorama, Kemeny (1992) propone la consolidación del campo de los estudios de vivienda para el abordaje de las relaciones sociales, políticas, económicas y culturales que directa o indirectamente intervienen en la actividad de planear, gestionar y usar las viviendas.

Al proponer lo anterior, Kemeny avanzó en la definición del concepto de "residencia" y lo consideró más apropiado que el de vivienda o el de hogar — los cuales recoge_- - puesto que permite una mirada más amplia del fenómeno, como parte de la estructura social. A juicio del autor, los análisis centrados en el concepto de "la vivienda” se enfocaban en su dimensión física en términos de producción, gestión y disposición, lo que resultaba insuficiente e implicaba la necesidad de enlazar tal dimensión con los factores sociales del hecho residencial. Estos podían sintetizarse en la dimensión espacial, en la que se destaca la organización espacial de la vivienda y su uso social, así como su integración en una organización espacial de mayor jerarquía.

Desde esta óptica, el concepto de residencia incorpora a la vivienda en sus contextos sociales y territoriales, al ampliar el foco de observación a sus componentes físicos y sociales.

La importancia de la propuesta de Kemeny para los estudios sociales de la vivienda puede sintetizarse en cuatro puntos: i) El traslado del objeto de investigación de la vivienda al hecho social de residir; ii) La incorporación del espacio al concepto de residencia, no solo en términos físicos, sino con una mirada ampliada hasta la localidad en donde se ubica; iii) El planteamiento de que la estructura social afecta el proceso de producción y consumo de la vivienda y también la "vivencia de la vivienda"; iv) La incorporación de dos perspectivas de análisis: la referida a las unidades familiares y la que se sitúa en un plano colectivo, con un papel estatal de especial importancia.

A partir de estos supuestos, Kemeny propone tres niveles de relación del hecho residencial con la estructura social (Cortés, 1995):

- La familia: según su composición, momento del ciclo familiar y estatus socioeconómico.

- La vivienda: de acuerdo con su tipo, sus características, facilidades, condiciones y su precio.

- La residencia: incluye el análisis de los servicios urbanos, de abastecimiento y de comercio.

Otras categorías conceptuales para el estudio de la vivienda como hecho social han sido producidas desde disciplinas como la Sociología Urbana. Lefebvre (1978), por ejemplo, propuso el 
concepto de "habitar", cuyo núcleo es la familia que habita una vivienda que, a su vez, se ubica en una sociedad. De allí que sea un concepto construido históricamente, enlazado con la estructura social que determina sus contenidos. En palabras de Cortés (1995): "El habitar se constituye por objetos que son producto de la actividad práctica de los hombres, por lo que debemos plantear el habitar como una función inherente a toda sociedad, a la que se añade una función significante" (p.136).

Con base en estos postulados, la exploración del habitar se produce en diferentes planos: el espacial, referido a la vivienda, localidad o región, área urbana o rural; el económico, que estudia el espacio del consumo, del ocio o el trabajo; el social, que aborda la estructura familiar, la clase social y la socialización; el político-institucional, referido al papel del Estado y la sociedad civil y, por último, el cultural, que indaga las ideologías $\mathrm{y}$ costumbres con respecto a la vivienda.

Estos dos - residencia y habitar- son conceptos capitales que abren un espectro para las investigaciones de la vivienda y las ubican en distintos ámbitos disciplinares, como el de Demografía, que concentrará la atención del siguiente apartado. En él se pretende reconocerlos principales argumentos que justifican el interés de la Demografía por la vivienda y la forma como la ha abordado.

\section{La demografía residencial}

El uso del término "demografía residencial" (housing demography) ha sido utilizado en el ámbito anglosajón para referirse a esta temática interdisciplinar (Gober, 1992), que no solo examina el comportamiento residencial de la población, sino también la formación, composición y características de los stocks de vivienda, los procesos de composición del hogar, la movilidad entre unidades residenciales y la ubicación en subáreas urbanas. Dichos análisis son concebidos dentro de un marco longitudinal de cambio del comportamiento residencial a lo largo de los cursos de vida individuales y se basa en las interconexiones existentes entre el stock de viviendas y su factor territorial (Myers, 1990).

Asimismo, la demografía residencial analiza la manera en que los comportamientos demográficos y las configuraciones familiares interactúan con los contextos residenciales y viceversa (Módenes y López-Colás, 2006).

El estudio de esta relación ha tenido las dificultades propias de un lugar de confluencia de dos disciplinas modernas como son la Demografía y la Urbanística, que en principio se plantearon como campos mutuamente excluyentes desde sus reflexiones conceptuales y los sistemas de información estadística que utilizaban.

Sin embargo, la multidisciplinariedad ha logrado permear los estudios de la población y la vivienda, al generar esfuerzos comunes frente a problemas reales que requieren la intervención de ambas ramas. A este respecto, el trabajo de Rossi, de 1955, Why families move, a study in the social psichology of the urban residential mobility, fue pionero en el intento de comprender el proceso del individuo en su elección de vivienda; de allí se deriva el término housing demogra$p h y$, que se mantiene hasta hoy en la literatura anglosajona.

Fundado en ello, se ha buscado la confluencia de enfoques y metodologías de las mencionadas disciplinas, con aportaciones tan importantes como la de Myers (1990), que ayuda a interpretar el papel protagónico de la población en las pautas de consumo residencial y a comprender cómo esta influye sobre el resto de elementos del sistema, 
por medio del acercamiento a factores como las interconexiones entre el mercado de la vivienda y la población residente, las dimensiones temporales mediante la exploración de los procesos longitudinales y las pautas espaciales de distribución de vivienda.

Otros autores tambiénhan avanzado en la identificación de los criterios racionales para la selección de los contextos residenciales de estudio (Bonvalet y Merlin, 1988; Lelièvre y Levy-Vroelant, 1992; Grafmeyer y Dansereau, 1998).

\section{Relación entre población y vivienda}

Los problemas prácticos que busca atender la Demografía Urbana tienen como trasfondo la relación de doble vía entre población y vivienda. Se sabe que las personas viven en hogares ${ }^{1} \mathrm{y}$ los hogares necesitan viviendas, pero la relación se intrinca al considerar que, a largo plazo, la oferta de viviendas tiende a aproximarse al número de hogares, aunque la vida útil del parque residencial no depende del ciclo de vida de los hogares (Clark yDieleman, 1996).

Por ello, autores como Mulder (2006) han desarrollado conceptualmente la interacción entre población y vivienda y tienen en cuenta que los cambios en la población afectan la demanda de vivienda y, a su vez, los cambios en la oferta residencial pueden estimular la formación de nuevos hogares. Esto da lugar a una interdependencia en la que la forma de tenencia adquiere un lugar fundamental.
En la primera de estas vías (la determinación de la población sobre la vivienda) se considera que el mercado de las viviendas se distingue del de otros bienes, en parte por estar altamente condicionado por las personas, sus trabajos, sus intereses, sus posiciones en la estructura social y la apropiación que hagan del espacio físico y social construido por la residencia y su entorno. En este punto, es preciso considerar que la producción de vivienda está sujeta a un extenso conjunto de leyes y regulaciones generadas en contextos de poder determinados. Además, en su compra no solo intervienen los compradores, sino otros actores sociales como los desarrolladores, los gestores y las instituciones financieras. De otra parte, existen numerosas implicaciones de este enlace en la generación de esquemas de segregación residencial socioeconómica y los fenómenos de especulación inmobiliarios, para lo cual abundan casos ejemplificadores como el bum residencial estadounidense o el sucedido en España durante la última década.

Mas la determinación social de la vivienda no solo se presenta por sus condiciones de mercado, pues las características demográficas de una sociedad también afectan el contexto residencial. De allí que tendencias como el envejecimiento de la población, el crecimiento de la potencial fuerza de trabajo, el decrecimiento del tamaño de los hogares y el incremento de la demanda de viviendas por parte de minorías étnicas sean algunos de los componentes a considerar en el estudio y la gestión del ámbito residencial (Hooimeijer, 1991).

El concepto de hogar puede tomar diferentes significados según el contexto y la disciplina desde la que se indague. Uno puede ser el censal, que habla de un conjunto de personas con o sin vínculos familiares, que comparte la misma vivienda y los mismos servicios y mantiene un gasto común para comer. Por su parte, desde la Psicología Ambiental, el hogar puede entenderse como una evolución del concepto de vivienda, que se presenta cuando la misma se modifica por los vínculos rituales que se viven en el ámbito familiar. En este orden de ideas, una vivienda se transforma en un hogar cuando sus residentes le imprimen un significado al espacio en que habitan (Aragonés y Sukhwani, 1994). En presente trabajo se toma como referencia el concepto de hogar desde una perspectiva sociológica, según la cual el hogar se refiere a la dimensión social de la familia en relación directa con su condición residencial. 
Del otro lado de la relación, entre los condicionantes que la vivienda ejerce sobre la población se pueden identificar cuatro tensiones (Mulder, 2006):

- Vivienda y migraciones: relaciona las condiciones de habitabilidad, tenencia y acceso a la vivienda que pueden favorecer o no la permanencia en determinadas zonas habitacionales$^{2}$; por ello, en los estudios sobre calidad de la vivienda y comportamientos residenciales, el estudio de la migración y de la movilidad residencial conlleva a la pregunta por la calidad de vida de quienes se movilizan, sus estrategias residenciales y su percepción sobre el lugar que habitan.

- Vivienda y formación de hogares: existen tres maneras de conformar nuevos hogares - las uniones, las emancipaciones y las separaciones_- La intensidad y el calendario de estos tres fenómenos son sensibles a las condiciones del mercado residencial, sobre todo al parque disponible y su calidad, por lo que logra potenciarlos o posponerlos según el grado de urgencia que implique el fenómeno.

- Vivienda y fecundidad: la disponibilidad de viviendas puede afectar el calendario de la cohabitación y del matrimonio, así como el de la fecundidad.

- Vivienda y mortalidad: cada vez menos abordada, pues su vigencia se reduce en la medida en que aumentan las condiciones de desarrollo. No obstante, en algunos países en vías de industrialización persiste la incidencia de las bajas condiciones de calidad y sanidad de las viviendas en la trasmisión de enfermedades letales.
En síntesis, la llamada "relación de doble hélice" entre vivienda y población tiene una profundidad innegable. Como se ha evidenciado, el lugar que adquiere dentro de este nexo la calidad de la vivienda y la percepción que los individuos tienen de ella es vital para comprender los comportamientos residenciales de los hogares y así proyectar contextos residenciales favorables.

\section{Temas de investigación de la demografía residencial}

Los problemas abordados por la demografía residencial son dinámicos y dependen de valores culturales que varían de una sociedad a otra. Por ello, los temas de investigación de la demografía residencial han ido variando con la evolución de la disciplina y según la escuela donde se realicen.

En 1984, Dieleman identificó algunos temas de investigación de interés, como las proyecciones de hogares y viviendas, el comportamiento del mercado de vivienda en la fase de reducción del ciclo de vida de los hogares y en los producidos a raíz de los divorcios, las variaciones regionales en el mercado de vivienda y la redistribución del mismo(Hooimeijer, 1991).

Hoy por hoy, muchas de estas preguntas han ido solucionándose a partir de avances conceptuales y metodológicos, aunque han salido a la luz nuevos interrogantes correspondientes a nuevas realidades sociales y demográficas. Según la clasificación de Hooimeijer (1991), los retos de la disciplina se concentran en tres campos fundamentales:

- Desarrollo de la vivienda: apunta a la generación de modelos multidimensionales que den cuenta de la dinámica de los hogares y de las necesidades cuantitativas y cualitativas de 
vivienda. En contextos de plena cobertura residencial, este aspecto tendría que ver con las causas del proceso de cambio de los hogares y en sus efectos en el sistema residencial. Mientras tanto, en contextos como el colombiano, en donde el déficit habitacional sigue siendo una realidad, esta línea incorporaría reflexiones sobre los mecanismos para vincular el factor demográfico en la planeación de proyectos de vivienda eficaces y satisfactorios.

- Demanda y movilidad residencial: se plantea estudiar la movilidad familiar en función de la edad, las condiciones laborales y la posición socioeconómica, entre otros componentes que permiten retratar la heterogeneidad en la demanda, anticipando que los movimientos de los hogares revelan sus preferencias en cuanto a los tipos de vivienda y sus atributos, y en últimas, sugieren el nivel de satisfacción que tienen con respecto a su residencia, en la concepción más amplia del concepto, que incluso tiene que ver con el costo que paga por ella. Se entiende la movilidad residencial y la escogencia de la vivienda como fenómenos de interacción de las características demográficas, socioeconómicas y de la vivienda de los hogares. Con todo, es necesario explorar la forma en que las condiciones de acceso a la vivienda determinan el proceso de su consecución y dan lugar a procesos que diferencian entre la elección y la preferencia. Esto último aplica de manera especial en contextos de alta desigualdad social, en donde el acceso a la vivienda está altamente segmentado.

- Relocalización de hogares y redistribución espacial: tiene como objetivo explorar la movilidad residencial como un hecho que implica una relación entre los diversos niveles territoriales e incluso incorpora dinámicas de movilidad intraurbana. De allí que esta línea exprese la importancia del territorio, su organización administrativa y las políticas que se ejecutan sobre él.

Estas líneas de investigación dejan entrever dos tendencias innovadoras en el campo de los estudios de vivienda, que permiten repensar las estrategias y los objetos de investigación. De un lado, muestran un fuerte énfasis en la identificación de necesidades residenciales cualitativas que complementan los estudios sobre déficit cuantitativo que tradicionalmente eran los que utilizaban herramientas demográficas como las proyecciones de hogares y población. De allí se desprende la segunda tendencia a resaltar, que corresponde a la inclusión de las percepciones de los hogares con respecto a su residencia y de los factores que determinan los comportamientos familiares frente a la misma, como aspectos relevantes para la producción de entornos de calidad. Según este esquema, la calidad no puede pensarse alejada de la satisfacción, pues la primera depende de componentes subjetivos afincados en las características particulares de los hogares; por ello, la correspondencia entre estos dos conceptos - calidad y satisfacción residencial — desde un punto de vista demográfico enriquece las formas de análisis de los hechos residenciales contemporáneos.

\section{Calidad de la vivienda y satisfacción residencial}

A continuación se hará un breve recuento de sus antecedentes y disyuntivas conceptuales y metodológicas y luego se mostrarán los principales resultados obtenidos en las investigaciones que los abordan.

Los estudios de calidad residencial surgen de la necesidad de medir las necesidades cualitativas existentes en ámbitos determinados, al considerar que las condiciones de la vivienda son un indicador fundamental de calidad de vida. De hecho, la mayoría de los indicadores de pobreza 
extrema que no se basan exclusivamente en el ingreso, contemplan condiciones estructurales y de servicios básicos residenciales. De manera más reciente, este tipo de estudios se ha utilizado como base de investigaciones acerca de la movilidad o satisfacción residencial, pues se asume que los individuos asumen como recursos para tomar decisiones residenciales las características de sus viviendas y las posibilidades de transformarlas ${ }^{3}$.

Sin embargo, no se ha alcanzado un consenso sobre los servicios mínimos con los que debe contar una vivienda ni los elementos estructurales que la clasifican como adecuada. Cada investigación, según su finalidad, incluye unas u otras variables, aunque puede apreciarse un criterio común: la selección de elementos que afecten de manera directa la salud de los individuos (Ayala y Navarro, 2004).

A pesar de la variedad de opciones, es posible hacer una categorización básica de los componentes de la vivienda que han sido contemplados en los estudios de calidad y ubicarlos en tres grupos: las instalaciones básicas (baños, tuberías y calefacción), la presencia de problemas estructurales (goteras en el techo, humedad, estado de marcos y ventanas) y hacinamiento (Ayala y $\mathrm{Na}$ varro, 2004).

Algunas tendencias innovadoras en este tema han abierto la factibilidad de incluir en los índices de calidad la valoración de las personas sobre su vivienda y datos acerca delas condiciones de habitabilidad del entorno, que también dan cuenta de la calidad residencial. No obstante, en la práctica se ha avanzado poco en estas propuestas y tampoco se ha logrado producir el mecanismo apropiado para agregar la información que demuestra la calidad de la vivienda con referencia a las características que la determinan. Este reto se comparte con los estudios de pobreza y ha sido de sus índices de donde ha tomado especial influencia. Dentro de los métodos desarrollados para dicho fin sobresalen dos vertientes: la de estudios que sintetizan la información con medios aritméticos o de ponderación y la de aquellos que utilizan técnicas de análisis multivariable.

Dentro de las primeras, una aportación inicial fue la de Townsend (1979) que seleccionó doce indicadores de condiciones residenciales y construyó un índice de privación basado en la suma aritmética de los bienes o servicios no presentes. La condición para elegirlos era su correlación con el ingreso. Este y otros estudios han mostrado que la adición aritmética no permite valorar el grado de urgencia o necesidad de los diferentes aspectos, por lo que estudios posteriores asumieron la tarea. Investigaciones como la de Desai y Shah (1998) procedieron a señalar el consumo modal de los bienes o servicios y dieron mayor peso a aquellas más extendidas en la sociedad y valoradas por los hogares como indispensables.

Aunque estos mecanismos siguen teniendo vigencia, los modelos multivariables son cada vez más empleados, porque ayudan a ganar en precisión, pese a que no resuelven por completo el tema de la objetividad en la selección de los parámetros.

Si bien la pregunta de la mayoría de estudios que abordan la calidad residencial es su grado y sus componentes, el principal aporte que hace la demografía residencial se halla en el ámbito de la identificación de variables que favorecen la tendencia a la baja calidad residencial. Así, se ha abordado el impacto de la ocurrencia de eventos como el matrimonio, el divorcio, la paternidad o 
el desempleo y el momento en que suceden estos hechos en las carreras residenciales de los hogares, con respecto a la calidad de sus viviendas (Davies Withers, 1998; Mulder y Wagner, 1998, 2001; Dieleman y Schouw, 1989; Feijten, 2005). La ocurrencia y el momento del ciclo de vida en el que los hogares experimentan acontecimientos de su carrera educativa, laboral y familiar tienen un lugar importante en el desarrollo de las carreras residenciales (Feijten yMulder, 2005).

Por su parte, el origen de los estudios de la satisfacción residencial está ligado a la revisión de las áreas más deprimidas de las ciudades e incluso llegan a convertirse en estudios sobre las condiciones mínimas que deben existir dentro y fuera de la vivienda para asegurar la calidad de vida de sus habitantes.

Con el paso del tiempo, estos estudios se han extendido para examinar distintos ámbitos territoriales como las grandes urbes (Miller, Tsemberis, Maliay Grega, 1980), los entornos suburbanos (Cutter, 1982), asentamientos de alto riesgo geográfico (Crull, 1994) y diferentes grupos poblacionales con características raciales, etarias y de género (Baillie y Peart, 1992). También se ha ampliado la funcionalidad de estos estudios que, además de estimar la calidad residencial, han buscado revelar los factores que explican la conducta del individuo hacia su residencia para predecir su conducta futura, así como desarrollar evaluaciones posteriores a la ocupación y a los ambientes residenciales todavía no ocupados (Amérigo, 1995).

Para Aragonés y Amérigo (1987), el concepto de "satisfacción residencial" se refiere a los criterios subjetivos, las actitudes o los afectos provocados por el hecho de residir en un lugar o en otro.

En palabras de Weidemann y Anderson (1985, p. 156) "Es la respuesta emocional a la vivienda, sentimiento positivo o negativo que los habitantes tienen por donde ellos viven. Como tal, es una representación global de las respuestas afectivas de la gente al ambiente socio-físico en el cual vive (Aragonés y Amérigo, 1987, p. 72).

Por su parte, la definición presentada por Gold (1980, citado en Amérigo, 1995) sostiene que el término se refiere a las gratificaciones o al placer que produce vivir en un ambiente concreto.

Gracias a la amplia producción académica frente al concepto de satisfacción residencial y pese a la problematización permanente del término, se ha llegado a cierto consenso en una definición más operativa que la entiende como el resultado de la comparación que la gente hace entre la percepción de la calidad de su ambiente residencial y sus estándares de lo que esta calidad debe ser. En conclusión, estaríamos hablando de la distancia que separa las aspiraciones de los logros respecto al ambiente residencial.

La demografía se vinculó al tema con Rossi (1955), quien planteó que las necesidades y aspiraciones de los hogares son dinámicas en función de su etapa en el ciclo de vida, ya que en cada una de estas se enfrentan diferentes requerimientos en el espacio físico y en el social. La heterogeneidad de estas necesidades y aspiraciones frente a la vivienda está dada por la diversidad de las trayectorias de vida individuales según características de género, raciales, familiares, etc. Con base en esta idea, se han desarrollado estudios que buscan reelaborar las carreras residenciales de los hogares en torno a su ciclo de vida (Lu, 1999).

Una nueva ola de estudios sobre la satisfacción residencial explora, además de las características físicas de las viviendas y las demográficas de los residentes, los factores de contexto que hacen que los vecindarios sean más deseables. Otra serie de estudios se ha enfocado en señalarlos factores subjetivos que intervienen en la percepción de satisfacción de los hogares, es decir, en 
considerar que las características inherentes a la situación demográfica, social y cultural de quienes habitan las viviendas son definitivas para establecer patrones de satisfacción y pueden originar prácticas residenciales diferenciadas en las que se exprese la carga de valor asignada a los rasgos físicos de la residencia.

A continuación se presentan dos esquemas de clasificación desarrollados por sendos investigadores, en los que, según diferentes criterios, se categorizan los aportes conceptuales realizados hasta el momento. El primer esquema es el de Amérigo (1995), quien presenta un panorama de las aportaciones de la Psicología agrupadas en tres categorías de acuerdo con el tratamiento que recibe la variable satisfacción residencial, entendida como:

1. Criterio de evaluación de la calidad de la vivienda, ubicada como una variable independiente. La relación individuo-ambiente residencial está mediada por el sistema de referencia social del individuo.

2. Un elemento predictor de conductas y, por ello, como desencadenante de intenciones de ajuste a un determinado ambiente. La movilidad residencial es el mecanismo de ajuste más común.

3. Fruto de la comparación entre la situación residencial real y la ideal que se realiza por la valoración de los aspectos físicos, a partir de las condiciones y las expectativas individuales sobre los patrones de calidad residencial.

Un segundo modelo conceptual es el construido por Hipp (2009) con base en cinco teorías que asignan diversos pesos a los factores personales (fundamento de las tres primeras teorías) y contextuales (cimiento de las dos últimas) en el estudio sobre las percepciones de la vivienda y pueden ser reseñadas así:
- Modelo de estratificación de lugares: afirma que las condiciones raciales y socioeconómicas de las personas afectan la satisfacción percibida, ya que limitan el acceso a vecindarios más deseables y les hacen sentir atrapados en el lugar donde deben residir. Esto se confirmó al observar que los residentes con mayores ingresos reportaron mayor satisfacción que los residentes con ingresos bajos. Sin embargo, en esta percepción de la satisfacción no se especifica cuál es el lugar de las condiciones reales de calidad (South y Crowder, 1997; Harris, 2001; Parkes, Kearns y Atkinson, 2002).

- Teoría de la comunidad de responsabilidad limitada: se enfoca en las características de los propietarios y propone que su grado de inversión social y económica para residir en el lugar afecta la satisfacción manifestada. Los datos han confirmado que los propietarios tienen mayor compromiso con el vecindario y menor voluntad de abandonarlo, por lo que presentan mayor satisfacción que los arrendadores (Janowitz, 1952; Harris, 2001; Lu, 1999).

- Modelo de la saciedad: parte del supuesto de que los residentes tienen necesidades y deseos particulares. Se sabe que, en la medida en que estas se vean saciadas, la satisfacción será mayor. En el tema de la vivienda, una de las principales necesidades es la cercanía a todo tipo de servicios públicos o urbanos, lo cual incluye nuevos elementos a contemplar (Galster, 1987; Sampson y Raundenbush, 2004).

- Teoría de la desorganización social: propone que los aspectos asimilables al mantenimiento del orden social — como la estabilidad residencial, la heterogeneidad racial y la calidad de las viviendas - afectan la formación de lazos sociales que permiten desarrollar el control social necesario para tratar la delincuencia y el crimen en el momento 
en que aparezcan; esto provee mayor satisfacción residencial ${ }^{4}$ (Skogan, 1986,1990; Skogan y Maxfield, 1981;Markowitz, Paul, Allen y Jianhong, 2001; Taylor, 1996; Woldoff, 2002; Warner, 2003).

- Teoría sistémica: plantea que la estabilidad residencial incrementa las interacciones entre residentes y ello genera mayores lazos con el vecindario (Adams, 1992).

En términos metodológicos, los estudios de satisfacción residencial se han enfrentado a la pregunta de cómo entender el entorno residencial, pues persisten las dificultades en la definición empírica de los límites entre la vivienda y el vecindario y, sobre todo, en la ubicación, dentro de estos dos ámbitos, de las dinámicas que suceden en las áreas semipúblicas intermedias, tan importantes en la vida urbana de ciertos grupos socioeconómicos. Es más: incluso el concepto de vecindario, discutido desde tan antiguas escuelas, sigue siendo motivo de debates, puesto que es una categoría definida individualmente con base en los significados asignados y no solo en componentes geográficos, lo cual dificulta su medición.

Otros interrogantes metodológicos se refieren a la oportunidad de la medición de los estudios de satisfacción y el procedimiento para ello. La pregunta sobre "cuándo" se funda en que la satisfacción residencial es un proceso dinámico, para cuya medición sería necesario contar con información retrospectiva o longitudinal. Aun así, la mayoría de los estudios realizados tienen una óptica transversal que impide identificar los cambios en las percepciones a partir de las intervenciones públicas o particulares o bien los cambios familiares en cuanto al grupo de referencia con el que se compara el hogar y con respecto al cual define sus expectativas.

Esta situación demuestra la dificultad que enfrentan los investigadores para contar con los datos que requieren, a sabiendas de que en buena parte de los casos es preciso utilizar la información arrojada por censos o encuestas realizadas por entidades estatales. Esto reduce el margen de acción de las investigaciones y circunscribe las posibilidades de la academia a la información producida muchas veces sin la perspectiva de aportar al análisis de ciertos fenómenos.

En los estudios que tienen mayores recursos se han diseñado instrumentos específicos para el acopio de información, lo cual ha enfrentado a los investigadores a la pregunta por el "cómo". En busca de las respuestas, se han diseñado diferentes instrumentos de medición en perspectiva correlacional, como el índice de calidad ambiental percibida, las escalas de satisfacción residencial y los cuestionarios de satisfacción (Amérigo, 1995).

\section{Resultados obtenidos}

El estudio de las variables que determinan la propensión a habitar viviendas de calidad y presentar satisfacción residencial ha sido uno de los principales intereses de los investigadores. Fruto de la revisión de algunos de los trabajos, a continuación se clasifican las variables relevantes en los tres niveles de análisis propuestos por Kemeny para la observación del fenómeno residencial. Se diferencian las variables según la medición de aspectos objetivos o de percepciones; se nota que, en la mayoría de los casos, solo uno de estos tipos es privilegiado en las investigaciones y pocas veces se intenta analizar su relación.

4 Según los datos obtenidos, las personas que percibían una alta criminalidad en sus vecindarios se declaraban menos satisfechas con sus viviendas. Además, se observó que la heterogeneidad racial reduce consistentemente la satisfacción, aunque este factor disminuye al controlar por renta y por tipo de hogar. 
Tabla 1.

Variables relevantes en el estudio de la calidad y satisfacción residencial

\section{Nivel/tipo Objetivo}

Edad

Ciclo de vida

Tipo de tenencia

Familia

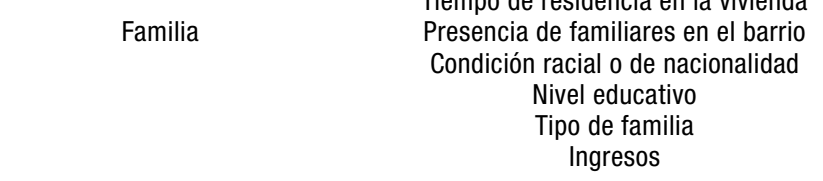

\begin{tabular}{|c|c|c|}
\hline Vivienda & $\begin{array}{c}\text { Hacinamiento } \\
\text { Unifamiliar/multifamiliar } \\
\text { Posesión de servicios públicos } \\
\text { Humedad } \\
\text { Problemas estructurales } \\
\text { Condiciones térmicas } \\
\text { Año de construcción }\end{array}$ & $\begin{array}{c}\text { Percepción de calidad de la vivienda/deterioro } \\
\text { Percepción de hacinamiento } \\
\text { Privacidad }\end{array}$ \\
\hline Residencia & $\begin{array}{c}\text { Existencia de espacios naturales } \\
\text { Oferta de infraestructura } \\
\text { Oferta de servicios urbanos }\end{array}$ & $\begin{array}{c}\text { Grado de mantenimiento del barrio } \\
\text { Aspecto estético del barrio } \\
\text { Nivel de ruido percibido } \\
\text { Administración del barrio } \\
\text { Sensación de seguridad } \\
\text { Relaciones vecinales } \\
\text { Homogeneidad social }\end{array}$ \\
\hline
\end{tabular}

Subjetivo
Esta categorización muestra varias tendencias: por un lado, se aprecia la fuerte concentración de investigaciones sobre las condiciones sociodemográficas de los hogares como determinantes de su relación con el espacio residencial.

En cuanto a la vivienda, se muestra que las mediciones han estado más enfocadas a la identificación de los problemas estructurales del parque residencial, que a las percepciones que tienen los hogares de este, aunque se han propuesto variables interesantes en este sentido.

En lo referido al entorno residencial, se evidencia que los estudios se han dirigido a la indagación por las relaciones sociales entre vecinos y otros asuntos como la percepción de seguridad del vecindario, pero han dejado de lado los rasgos físicos que lo constituyen.

Lo anterior indica que el análisis de los factores subjetivos que determinan la percepción de satisfacción con la vivienda, sea por sus características físicas internas o por la composición y los servicios ofrecidos en el entorno, sigue siendo un campo de trabajo por explorar. La urgencia de desarrollar avances investigativos en esta materia es manifiesta, dado que esto favorecería el aprendizaje sobre las prácticas y preferencias residenciales de los hogares según sus características, lo que permitiría hacer más aplicables los conocimientos sobre la existencia de elementos culturales en la apropiación de los espacios de vivienda. De allí que se pudiera profundizar en los puntos clave para generar proyectos residenciales acordes con las características de quienes los habitarán, para favorecer la eficacia de la inversión de recursos públicos y privados.

\section{Conclusiones}

Este recorrido por los enfoques del estudio de la demografía residencial ayuda a comprender la profundidad de las implicaciones de considerar el hecho residencial como un campo que supera las tradicionales concepciones de la vivienda 
como un hecho físico y objetivo. Esta forma de abordaje del fenómeno residencial, brevemente explorado en contextos como el colombiano, señala una serie de relaciones conceptuales que vale la pena desarrollar a profundidad en nuestro medio. A continuación se indican por lo menos tres:

Población-vivienda: relación desarrollada de manera extensa como sustento de la demografía residencial. Va desde la reflexión sobre el mercado inmobiliario hasta la forma en que se apropia y se dispone el espacio para construir en él la vida familiar, según las condiciones propias del hogar y de las posibilidades que brindan los espacios físicos internos y externos a la vivienda.

- Vivienda-entorno: recoge la relación entre las distintas esferas - micro, meso y macrodel hecho social de residir. En este sentido, explora la vinculación entre lo que sucede al interior de la vivienda y lo que pasa en los contextos externos, que van desde el barrio o el vecindario hasta el ámbito urbano en su conjunto. Esta tensión surge cuando se reconoce que el hecho de residir no solo se localiza en la vivienda y que el entorno influye también en la satisfacción residencial, por lo que deberían constituir la medida de calidad de la misma.

- Realidad-percepción: esta relación se asemeja a la trazada entre calidad y satisfacción, en la que se revisa la diferencia entre lo esperado y lo obtenido. Esta complementariedad entre lo objetivo y lo subjetivo también permite captar matices en los componentes de las necesidades residenciales cualitativas y en las formas de medir la satisfacción residencial.

\section{Bibliografía}

Amérigo, M. (1995). Satisfacción residencial: un análisis psicológico de la vivienda y su entorno. Madrid: Alianza Universidad.
Aragonés, J. I. y Amérigo, M. (1987). Satisfacción residencial: Un concepto de calidad de vida. Documentación Social (67), 133-154.

Aragonés, J. I. y Suhkwani, S. (1994). La vivienda como escenario de conducta y símbolo de la identidad. En E. Wiesenfeld (comp.), Contribuciones iberoamericanas a la Psicología Social (pp. 57-89). Caracas: Universidad Central de Venezuela.

Atkinson, A. B. (2003). "Multidimensional Deprivation: Contrasting Social Welfare and Counting Approaches. The Journal Inequality, 1(1) 51-65.

Ayala, L. y Navarro, C. (2004). Multidimensional Indices of Housing Deprivation with Application to Spain. Papeles de trabajo del Instituto de Estudios Fiscales, 12(4), 1-31.

Baillie, S.T. y Peart, V. (1992).Determinants of Housing Satisfaction for Older Married and Unmarried Women in Florida. Housing and Society, 19(2), 101-116.

Bonvalet, C. y Merlin, P. (eds.) (1988). Transformation de la famille et habitat. París: L'Harmattan.

Brandolini, A. y D'Alessio, G. (27 de agosto de 2000). Measuring Well-Being in the Functioning Space. Conferencia presentada en 26th General Conference of The International Association for Research in Income and Wealth, Cracow.

Chakravarty, S. R. y D'Ambrosio, C. (2003). The Measurement of Social Exclusion. DIW (Documento de discusión). Recuperado de EconPapers. (RePEc:diw:diwwpp:dp364).

Clark, W. y Dieleman, F. (1996). Housing and Households. Choice and Outcomes in the Housing Market. Nueva Jersey: Centre for Urban Policy Research.

Cortés, L. (1995). La cuestión residencial. Madrid: Fundamentos. 
Crull, S. R. (1994). Housing Satisfaction of Households at Risk of Serious Housing Problems. Housing and Society, 21(2), 62-75.

Cutter, S. (1982). Residential Satisfaction and the Suburban Homeowner. Urban Geography, 3(4), 315-327.

Davies Withers, S. (1998). Linking Households Transitions and Housing Transitions: A Longitudinal Analysis of Renters. Environment and Planning $A$, 30(4), 615-630.

Desai, M. y Shah, A. (1988).An Econometric Approach to the Measurement of Poverty, Oxford Economic Papers, 40(3), 505-522.

Dieleman, F. M. y Schouw, R. J. (1989). Divorce, Mobility and House Demand. European Journal of Population (5), 235-252.

Dureau, F. (1999).Les mobilités à géométrie variable des habitants de Bogota.Espace, Populations, Sociétés (2), 329-344.

Dureau, F. (2002).Les systèmes résidentiels: concepts et applications. En J-P. Lévy y F. Dureau (dir.), L'accès à la ville. Les mobilités spatiales en questions (pp. 355-382). París: L'Harmattan.

Dutta, I., Pattanaik, P. K. y Xu, Y. (2003). On Measuring Deprivation and the Standard of Living in a Multidimensional Framework on the Basis of Aggregate Data. Economica, 70(278), 197-221.

Feijten, P (2005).Union Dissolution, Unemployment and Moving out of Homeownership. European Sociological Review, 21(1), 59-71.

Feijten, P. y Mulder, C. (2005). Life Course Experience and Housing Quality. Housing Studies, 20(4), 571-587.

Galster, G. (1987). Homeowners and Neighborhoods Reinvestment. Duke: Press Policy Studies.
Gober, P. (1992).Urban Housing Demography. Progress in Human Geography, 16(2), 171-189.

Grafmeyer, Y.y Dansereau, F. (eds.) (1998).Trajectoires familiales et espaces de vie en milieu urbain. Lyon: Presses Universitaires de Lyon.

Harris, D. (2001). Why are Whites and Blacks Averse to Black Neighborhoods? Social Science Research, 30(1), 100-116.

Hipp, J. R. (2009). Specifying the Determinants of Neighborhood Satisfaction: A Robust Assessment in 24 Metropolitan Areas. Social Forces, 88(1), 395-442.

Hooimeijer, P. (1991). Towards Spatial Demography of Housing. En C. Hazeuy G. Frinking (eds.), Emerging Issues in Demographic Research (pp. 281291). Amsterdam: Elsevier Science publisher.

Janowitz, M. (1952). The Community Press and Urban Setting. Glencoe: Free Press.

Kemeny, J. (1992). Housing and Social Theory. Londres, Nueva York: Routledge.

Lefebvre, H. (1978). El derecho a la ciudad. Barcelona: Península.

Lelièvre, E. y Levy-Vroelant, C. (eds.) (1992). La ville en mouvement: Habitat et habitants. París: L'Harmattan.

Lévy, J. P. (2003). Population Patterns and Household Trajectories in the Residential Mileu: The Example of the Seine-Saint-Denis. Population, 58(3), 323-357.

Lévy, J-P. (1998). Habitat et habitants: position et mobilité dans l'espace résidentiel. En Y. Grafmeyer y F. Dansereau (eds.), Trajectoires familiales et espaces de vie en milieu urbain (pp. 153-180). Lyon: Presses Universitaires de Lyon.

Lévy, J-P. (2002). The Population Make-Up of Residential Areas: Structure and Changes 
(Douai, France).Journal of Housing and the Built Environment (17), 293-319.

Lu, M. (1999). Determinants of Residential Satisfaction: Ordered Logit Vs. Regression Model. Growth and Change, 30(2), 264-287.

Markowitz, F.E., Paul, B., Allen, L. y Jianhong, L. (2001). Extending Social Disorganization Theory: Modeling the Relationship between Cohesion, Disorder and Fear. Crimonology, 39(2), 293-319.

Miller, F., Tsemberis, D., Malia, G. P. y Grega, D. (1980). Neighborhood Satisfaction among Urban Dwellers. Journal of Social Issues, 36(3), 101-117.

Módenes, J. y López-Colás, J. (2006). Población y vivienda en España: sistema residencial a la luz del Censo del 2001. Papers de demografia, 95(3), 655-672.

Mulder, C. (2006). Population and Housing: A Two-Sided Relationship. Demographic Research, 15(13), 401-412.

Mulder, C. H. y Wagner, M. (1998). First-Time Homeownership in the Family Life Course: A West German-Dutch Comparison. Urban Studies, 35(4), 687-713.

Mulder, C. H. y Wagner, M. (2001). The Connections between Family Formation and FirstTime Home Ownership in the Context of West Germany and the Netherlands. European Journal of Population, 17(2), 137-164.

Myers, D. (1990). Housing Demography. Londres: The University of Wisconsin Press.

Myers, D. y Lee, S. W. (1996). Immigration Cohorts and Residential Overcrowding in Southern California. Demography, 33(1), 51-65.

Parkes, A., Kearns, A. y Atkinson, R. (2002). What Makes People Dissatisfied with Their Neighborhoods? Urban Studies, 39(13), 2413-2438.
Rossi, P. H. (1955). Why Families Moves: A Study in the Social Psychology of Urban Residential Mobility. Glencoe: Frees Press.

Sampson, R. y Raundenbush, S. (2004). Seeing Disorder: Neighborhood Stigma and the Social Construction of "Broken Windows". Social Psychology Quarterly, 67(4), 319-342.

Skogan, W. G. (1986). Fear of Crime and Neighborhood Change. En A. J. Reiss y M. Tonry (eds.), Communities and Crime (pp. 203-230). Chicago: University of Chicago Press.

Skogan, W. G. (1990). Disorder and Decline: Crime and the Spiral of Decay in American Neighborhoods. Los Angeles: Free Press.

Skogan, W. G. y Maxfield, P. (1981). Copying With Crime: Individual and Neighborhood Reactions. Beverly Hills: Sage.

South, S. y Crowder, K. (1997). Escaping Distressed Neighborhoods: Individual, Community, and Metropolitan Influences. American Journal of Sociology, 102(4), 1040-1084.

Taylor, J. (1996).Neighborhood Responses to Disorder and Local Attachments: The Systemic Model of Attachments, Social Disorganization and Neighborhood Use Value. Sociological Forum, 11(1), 41-74.

Townsend, P. (1979). Poverty in the United Kingdom. Harmondsworth: Penguin.

Warner, B. (2003). The Role of Attenuated Culture in Social Disorganization Social Theory. Criminology, 41(1), 73-97.

Woldoff, R. (2002). The Effects of Local Stressors on Neighborhood Attachments. Social Forces, 81(1), 87-116. 